\title{
MAPublisher - Little Known Tips and Tricks
}

The following is a summary of the brief presentation given at the NACIS XXI conference in Portland, OR on October 3, 2001.

A s many of those present were either already users of MAPublisher or were familiar with it in one way or another, it was decided that a short presentation outlining a few useful tips and tricks would serve everyone well. All data files used in these examples can be found at ftp:/ / $\mathrm{ftp}$.avenza.com/pub/misc/NACIS_XXI_files.zip

1. Importing Multiple GIS Data Files Individually to Scale to a Predefined Page Size - When a GIS data file is imported to Illustrator or FreeHand using MAPublisher, MAPublisher calculates a scale for the map layer which will best fit the data onto the current page. Problems can result when layers of different extents, which cover roughly the same geographic area, are imported separately, such that the layer with the greater extents falls outside the Illustrator or Freehand page. The trick to making sure that all layers import and line up properly and that no data falls outside the page is to import the layer with the largest extents first. Subsequent layers can then be imported using the "Same as" option with the initial layer selected. To demonstrate this, create a new page in Illustrator or FreeHand using the landscape orientation. Be sure to set the rulers to 0,0 at the lower left corner. This example uses the railroad and county layers for King County, WA. The railroads do not fully extend to the county boundary so if the rail file is imported first MAPublisher will calculate a scale based on the rail layer. Then, when the county boundary file is imported, portions of it will fall outside the page in order to ensure that it registers with the rails layer. Start by importing the kingWArails.shp file using the "Defaults" option. The file will be imported and the railroad lines will extend to the edge of the page. Now import the kingWAboundary.shp and select the "Same as" option, referencing the kingWArails layer. The boundary file will import at a scale that matches the rails layer but in so doing the county boundary will fall outside the page. This is the situation we wish to avoid. Now start a new page and repeat the above procedure importing the boundary file first. Notice that after the railroad layer is imported both layers fall inside the page and are registered to each other as desired. Note that with MAPublisher 4.0 multiple data files can be imported at one time such that the scale is set by the file with the greatest extents.

2. Importing With a Grain - Frequently it is desirable or necessary to "thin" a GIS file upon import. This is particularly the case with very complex area files that exceed the points-per-path limits set by Illustrator and FreeHand or with large files that exceed your computer's processing ability. In this example we will see how the MAPublisher "Grain" function is used to import a complex polygon layer. This example uses the outline of Norway (norway.mif) file. Start by creating a new page in landscape orientation. Be sure to set the 0,0 point

\author{
Ted Florence \\ Avenza Systems, Inc. \\ ted@avenza.com
}

"Problems can result when layers of different extents, which cover roughly the same geographic area, are imported separately, such that the layer with the greater extents falls outside the Illustrator or Freehand page."

"Frequently it is desirable or necessary to 'thin' a GIS file upon import." 
". . you may desire to plot some points on your map using world coordinates where such a point GIS file is not available." to the lower left corner. Now import the norway.mif file using the "Defaults" function and without entering a grain value. The file will import, however you should notice there has been a truncation of the line work of the main Norway polygon resulting in two points being joined by a straight line and a number of points not being joined at all. This is obviously incorrect and can be addressed by re-importing using the "Grain" function. Rename the "norway" layer to "norwaynograin" in your layers palette. Now re-import the norway.mif file. If you are using Illustrator be sure to check the "grain" box before selecting the norway.mif file. After the norway.mif file appears in the import list you will be able to enter a value for the grain in the row corresponding to this file. Enter a value of 0.0225 in the cell corresponding to the grain value for the norway.mif file. Now complete the import using the "Defaults" option and the file will import correctly and overlay the original "no-grain" import. You can now compare the difference between the two import processes by selecting one of the Norways and moving it to a blank portion of the page so both outlines can be seen at the same time. You can also open the norway-both.ai and norway-compare.ai files that have been included with the accompanying data.

3. Creating Your Own ASCII Point File - From time to time, you may desire to plot some points on your map using world coordinates where such a point GIS file is not available. In this example we will see how to create a point file using a text editor that will enable points to be placed in their correct geographic locations on an existing FreeHand or Illustrator MAPublisher map. To start, open the westcoastUSA.ai file or import the westcoastUSA.mif file to a new page. This file will form the base for the points we wish to plot. In this case we will create a file that will place points for 3 major cities on the US west coast. Now open Notepad, SimpleText or another text authoring program and create a new document and enter the following 4 lines of text:

\section{Lat,Long,City \\ 45.565,-122.86,Portland \\ $47.45,-122.38$, Seattle \\ 37.70,-122.49,San Francisco}

The first line is optional. We have included it in this case and will use it as the header line when we import the file. As the base map (westcoastUSA.mif) is in lat/long coordinates we will use lat/long values to plot our points. The Lat value is the latitude and the Long value is the longitude. All other values will be used to construct the attribute table for the points. In this case there is only one, City, however you can have as many as you'd like. An important thing to remember here is to perform a hard carriage return after the last line. Save the file as cities.txt or some other reasonable name. Now choose a font and point size of 10 and open the MAPublisher Import Points dialog. Select the text file you just created as the file to import and complete the fields in the rest of the dialog by choosing the Long column for the $X$ coordinates, the Lat column for the $\mathrm{Y}$ co-ordinates and a symbol to use for the points. Select the "Same as" option under Page Scaling Information and reference the westcoastUSA layer. Check the "use first line as header" box if your file has a header line like ours does. Now click OK and the points will 
be imported to a new layer called cities with the points placed according to the lat/long values we used in the text file. The attributes table will also be constructed and can be viewed using the "Show Map Attributes" function. Files such as our cities.txt file can also be made using common spreadsheet programs such as Microsoft Excel using the export as comma-delimited option.

4. Dropping Columns - Removing unnecessary data columns from the MAPublisher attributes table is a very easy and handy way of improving the overall performance of your map file. GIS data files often include many columns of data that are entirely un-necessary for cartographic construction and end up causing slow "Select by Attribute" and "Draw Legend" routines and can make files larger than need be. For example, the US counties file (counties.shp) contains 12 columns of data of which we may only need the single column that contains the county name. Import the counties.shp file to a new Illustrator or FreeHand page, select one or two features, and open the MAP Attributes window to view all the columns of data. Now let's remove a few columns that we don't need. Illustrator users should click the MAP Columns tab, select one or more of the column names they wish to delete and then click on the trash can symbol at the bottom right of the window. FreeHand users must open the "Show MAP Columns" window, select one or more columns to delete and then select Options->Delete column within the "Show MAP Columns" window. Now try this again with the az_deci(partial).txt point file or with your own data file.

5. Joining Arcs - Map files can also be made smaller and easier to work with by joining similarly attributed linear features together to form single longer lines. The end result is a new line layer with much fewer line features, each with only a single attribute column, hence faster redraws, searches and selections. Joining arcs is also useful prior to labeling using MAPublisher's "Feature Text Label" tool. Try this using the toronto-streets.mif file. After importing this file open the MAPublisher Selection Statistics window and notice that the toronto-streets layer has 1197 lines on it and using the MAP Attributes window notice the number of data columns for each line feature. Now using the MAPublisher "Join Arcs" function, join all the lines by selecting toronto-streets as the layer, Street as the column and entering 0.0001 as the proximity value. Name the output layer torontostreets-joined. After the join is complete select the toronto-streetsjoined layer and using the MAPublisher Selection Statistics window you should notice that there are now only 289 lines present yet the map looks identical. The MAP Attributes window will reveal only a single attribute column for that layer. Now the original unjoined layer can be deleted if no longer required.

6. MAP Tagger Tool vs Feature Text Label - MAPublisher offers two methods of labeling using the attributes table of an imported GIS file. The "MAP Tagger" tool is used for labeling individual features of a map by clicking on each feature, whereas the "Feature Text Label" function is used for labeling multiple items at a time. To demonstrate the appropriate usage of each tool, start by importing the quad250.e00 file to a new document. Now after choosing an appropriate font and point size select the "MAP Tagger" tool from the tools palette and choose an appropriate column

\author{
"Removing unnecessary data \\ columns from the MAPublisher \\ attributes table is a very easy \\ and handy way of improving \\ the overall performance of your \\ map file."
}

"Map files can also be made smaller and easier to work with by joining similarly attributed linear features together to form single longer lines."
"MAPublisher offers two methods of labeling using the attributes table of an imported GIS file." 
"One of the most overlooked functions in MAPublisher 4.0 is its ability to generate an accurate index for labeled features."

"A little known feature of MAPublisher's indexing function is the ability to generate an index from an un-georeferenced or non-MAPublisher created map." for labeling. Then begin clicking within each of the polygons and place a few labels. As you will quickly imagine, labeling this map that way or perhaps one with 100's or even 1000's of elements can be a tedious and time-consuming process. Now delete the labels you have just placed. Select either all the features on the layer or only a few that you wish to label and select "Feature Text Label" from the menu of MAPublisher functions. Complete the entries in the ensuing dialog and after clicking OK all the selected features will be labeled at once. This quickly illustrates the power of "Feature Text Label" over "MAP Tagger" but what about the converse? Start a new document and import the toronto-streets.mif file. This is an unjoined line file and can be used to demonstrate when the "MAP Tagger" tool is more appropriate. Using the "Feature Text Label" function on such a layer will result in multiple labels being placed for each road, given that each road is comprised of several segments, each with the same name. However, one can quickly label the roads using the "MAP Tagger" tool. In such a case only the lines that have been clicked on will receive labels. This affords greater precision in labeling files of this nature by allowing you to click only the segments you want labels on.

7. Make Index - One of the most overlooked functions in MAPublisher 4.0 is its ability to generate an accurate index for labeled features. Try this by opening the world.ai file or importing the world.mif file. Now label a few countries using the "MAP Tagger" tool. Create a new layer and name it 'grid'. Use the MAPublisher "Add MAP Parameters" function to assign a scale and coordinate system to the grid layer using the "Same as" world layer function. Open the MAPublisher "Grid Generator" dialog and enter the following values:

- check the "generate index labels" box and select the options you desire for the grid labeling for index purposes

- select page units

- set columns to 11 and rows to 6

- set UR X to 792 and UR Y to 400

- "cell size" next to the "Calculate" button and click the calculate button

Now click OK and the grid will appear complete with alphanumeric labels along its axis or within each cell depending upon the option you chose earlier. Now open the MAPublisher "Make Index" dialog. Select "Text Objects" and set the layer to world and grid layer to grid. Select the output options you desire for the resulting index formatting and click "Save As" and name your index text file when prompted. Your index will now be saved to the file and location you just named and can be copied and pasted or placed into your map document.

8. Indexing a Non-MAPublisher Map - A little known feature of MAPublisher's indexing function is the ability to generate an index from an un-georeferenced or non-MAPublisher created map. This can be very useful if you wish to index a map you made before you began using MAPublisher. To illustrate this, open the index2.ai file or simply create a basic new document containing a few lines or polygons and associated text elements or labels. Now "Add MAP Parameters" to the layer you wish to index, using all the default values in 
the "Add MAP Parameters" dialog. Then select all the text you wish to be indexed and "Add Text Defaults" to it. Then, by following the steps outlined in the previous example you will create an index for the index2.ai file or whichever one you happened to create in this example.

Questions regarding this presentation, the data files or any other MAPublisher-related issues can be sent to support@avenza.com. 